\title{
A Inteligência Artificial e seu impacto na construção do Direito como norma de
} decisão

\author{
Artificial Intelligence and its impact on the construction of Law as norm of decision \\ La Inteligencia Artificial y su impacto en la construcción del Derecho como regla de decision
}

Recebido: 22/04/2021 | Revisado: 01/05/2021 | Aceito: 01/07/2021 | Publicado: 04/07/2021

Francisco Junior de Oliveira Marques
ORCID: https://orcid.org/0000-0002-2684-6499
Universidade de Fortaleza, Brasil
E-mail: sssmarquez@ @otmail.com
Rafael Marcílio Xerez
ORCID: https://orcid.org/0000-0001-7472-6822
Universidade de Fortaleza, Brasil
E-mail: rafaelmx@unifor.br
José Cândido da Silva Nóbrega
ORCID: https://orcid.org/0000-0002-0976-3763
Universidade Federal de Campina Grande, Brasil
E-mail: jcandidosn@uol.com.br

\section{Resumo}

O presente manuscrito visa analisar o impacto da inteligência artificial na construção do Direito como norma de decisão. O diálogo entre a inteligência artificial e o Direito, dessa forma, é indispensável averiguar a definição de inteligência artificial, bem como realizar uma investigação minuciosa sobre o processo de construção da norma decisória, tendo como referencial Friedrich Müller. Nesta perspectiva, o presente artigo, por meio da pesquisa explicativa, de natureza qualitativa, método dedutivo, coleta de dados bibliográfica e documental, desenvolveu-se de modo a tecer considerações acerca da cibercultura, sobretudo no tocante ao suporte tecnológico intelectual capaz de dar respostas automáticas para as várias áreas do conhecimento humano, tornando-o mais acessível, transparente e econômico. Por fim, conclui-se diante dos resultados que a inteligência artificial, não alcança reproduzir habilidades cognitivas de alta inteligências para concretizar a norma de decisão nos moldes da inteligência humana, contudo, pode-se pensar na sua influência à luz de modelos de informação patronizada por algoritmos.

Palavras-chave: Inteligência artificial; Direito; Norma de decisão.

\begin{abstract}
The present manuscript aims to analyze the impact of artificial intelligence in the construction of Law as a decision rule. The dialogue between artificial intelligence and law, in this way, it is essential to investigate the definition of artificial intelligence, as well as conducting a thorough investigation of the decision-making process, using Friedrich Müller as a reference. In this perspective, this article, through explanatory research, of a qualitative nature, deductive method, collection of bibliographic and documentary data, developed in order to make considerations about cyberculture, especially with regard to intellectual technological support capable of providing answers for the various areas of human knowledge, making it more accessible, transparent and economical. Finally, it is concluded in view of the results that artificial intelligence, does not manage to produce cognitive abilities of high intelligences to materialize the decision standard along the lines of human intelligence, however, one can think about its influence in the light of models of patronized information by algorithms.
\end{abstract}

Keywords: Artificial intelligence; Right; Decision standard.

\section{Resumen}

El presente manuscrito tiene como objetivo analizar el impacto de la inteligencia artificial en la construcción del Derecho como regla de decisión. El diálogo entre inteligencia artificial y derecho, de esta forma, es fundamental para investigar la definición de inteligencia artificial, así como realizar una investigación exhaustiva del proceso de toma de decisiones, tomando como referencia a Friedrich Müller. En esta perspectiva, este artículo, a través de una investigación explicativa, de carácter cualitativo, método deductivo, recolección de datos bibliográficos y documentales, se desarrolló con el fin de realizar consideraciones sobre la cibercultura, especialmente en lo que se refiere al soporte tecnológico intelectual capaz de dar respuestas para las distintas áreas. del conocimiento humano, haciéndolo más accesible, transparente y económico. Finalmente, se concluye a la vista de los resultados que la inteligencia artificial, no logra producir habilidades cognitivas de altas inteligencias para materializar la norma de decisión en la línea de la inteligencia humana, sin embargo, se puede pensar en su influencia a la luz de modelos de inteligencia humana. información patrocinada por algoritmos.

Palabras clave: Inteligencia artificial; Derecha; Estándar de decisión. 


\section{Introdução}

O presente artigo analisa o impacto das máquinas dotadas de inteligência artificial na construção da norma decisória. No primeiro item, será abordado o conceito de máquinas dotadas por inteligência artificial, sua origem, características, limites e possibilidades. No segundo item, debruçar-se-á sobre o processo de construção da norma decisória a partir da teoria estruturante do direito de Friedrich Müler. Finalmente, tendo analisado conceitos e processos da inteligência artificial (IA) e do Direito, o terceiro item abordará as reais possiblidades de diálogo entre esses campos do conhecimento.

A metodologia é bibliográfica, elaborada a partir de material publicado em livros, revistas e pesquisa em bases eletrônicas; bem como documental, mediante consulta a legislação. Quanto aos objetivos, a pesquisa é explicativa, pois identifica e analisa o fenômeno dos possíveis impactos da IA na prática do Direito. Quanto à abordagem ao problema apresentado, faz-se um estudo qualitativo. Finalmente, a pesquisa é de natureza teórica.

A principal motivação para escrever este artigo é oferecer uma visão realista e desmitificada da tecnologia corrente da IA, que certamente contrasta com uma visão futurista das capacidades ou habilidades da IA. Não se trata de especular os efeitos ou influências da IA sobre a prática do Direito numa sociedade próxima ou longínqua. Ainda que se reconheça a relevância de tais discussões, o caminho que se faz aqui foca nas políticas de eficiência, acesso e baixo custo do Direito, pensadas a partir das novas tecnologias.

\section{A Relevância do Tema e sua Problematização}

A predição de decisões judiciárias, a análise automática de jurisprudência, a resolução de litígios online, enfim, o início da revolução tecnológica no Direito é um tema relevante e urgente para os atores do Direito contemporâneo. Nos últimos anos, o crescimento de investimentos em empresas de tecnologia jurídica permaneceu num standard baixo, contudo, em 2018, houve uma explosão econômica. A Forbes (Privovarov, 2019) revela que, em 2016, foram investidos na indústria de tecnologia jurídica o valor de U\$ 224 milhões e, em 2017, de U\$ 233 milhões. Em 2018, as cifras foram de U\$ 1.6 bilhões, com um crescimento de $713,7 \%$ com relação ao ano anterior. Já os primeiros valores parciais de 2019, apresentados pela bloomberglaw (Mcevoy, 2019), chegaram aos U\$ 1.2 bilhões.

A e-Discovery é o exemplo que mais cresceu no mercado da indústria de tecnologia jurídica, sobretudo entre os países de sistema jurídico da Common law. Esta plataforma virtual é um serviço eletrônico de busca, localização e obtenção de informações nos processos judiciais, investigações, e oferece aos advogados ajuda, poupando-lhes tempo com maior precisão na busca de casos judiciais adequados as suas necessidades. Outro projeto importante no mercado da justiça online é a Cyberjustice, da Faculdade de Direito de Montreal (Umontreal). Seu crescimento e importância com relação à acessibilidade e redução de custos da Justiça, o transformou no maior referencial de tecnologia digital do Direito.

No Brasil, após a Resolução n 125/2010, sobre Política Nacional de tratamento adequado de conflitos, o Judiciário passou a dispor sobre métodos eletrônicos de solução de conflitos. Emergiram as primeiras plataformas no Brasil destinadas a resolver litígios, movendo o sistema judiciário da era off-line analógica para o mundo digital da Revolução 4.0 (Rosa \& Spaler, 2019). A partir desse início incipiente, o mercado jurídico abriu-se para as empresas de Online Dispute Resolution (ODR) e, em 2017, nasceu a Associação Brasileira de Lawtech e Legaltech (AB2L), que conta com quase 150 startups jurídicas, provedoras de serviços no seguimento.

O crescimento exponencial demonstra a força disruptiva desse novo mercado, que surge em bom momento para ajudar a solucionar os altos números de demandas na Justiça. Conforme dados do CNJ (28/08/2019), o Brasil tem a marca de 78,7 milhões de casos sem solução em 2018. Ao comparar o número de processos em andamento e o universo de 210.665,164 milhões de habitantes no Brasil (IBGE, 2019), pode-se concluir o alto nível de litigância da sociedade e, consequentemente, a necessidade de oferecer aos cidadãos métodos para agilizar a solução das demandas, com redução de tempo e custos. 
A cibercultura transforma a maneira de pensar, a forma de consciência e a memória coletiva do Direito, com sua vasta documentação registrada relativa a jurisprudência, leis e práticas intelectuais. Considerando que os computadores e, especialmente, a cibercultura é um suporte tecnológico intelectual capaz de dar respostas automáticas para as várias áreas do conhecimento humano, tornando-o mais acessível, transparente e econômico. Nesta esteira, pergunta-se: pode a máquina dotada de inteligência artificial construir normas decisórias? A partir dessa pergunta será apresentado um itinerário que dialoga com Direito e IA.

\section{As Máquinas Dotadas de Inteligência Artificial}

As grandes mutações do saber deram-se a partir do desejo do homem de controlar a realidade. As ciências analíticas da natureza preponderaram durantes os últimos séculos. Contudo, o paradigma não se sustentou, e a antropologia pluridimensional (Bôas Filho, 2010), bem como a ambição pela interconectividade, voltaram com toda força, agora em um cenário de virtualização digital.

A humanidade apenas iniciou essa etapa evolutiva de virtualização pós-moderna no ciberespaço. A primeira descrição gravada das interações sociais que poderiam ser viabilizadas através de redes deu-se na série dos escritos de J.C.R. Licklider, em agosto de 1962, a partir do conceito galatic network. Em 1966, Lawrence G. Roberts apresentou à agência norte-americana DARPA (Advanced Research Projects Agency), o projeto ARPANET para desenvolvimento de uma rede de computadores. Em setembro de 1969, a Bolt Beranek and Newman (BBN) instalou o primeiro Interface Message Processors (IMP's), no primeiro computador host conectado (Leiner, 2019). Esta primeira rede de computadors foi desenvolvida de forma fechada, inicialmente com finalidade militar e, posteriormente, conectando instituições de pesquisa. $\mathrm{O}$ uso da rede somente foi aberto para a sociedade civil no final da década de 1980.

Depois de aproximadamente três décadas, Pierre Levy (2011, pp. 63-130) reconhecer três traços relevantes e originais do ciberespaço em relação à história da cultura humana. A primeira destas características é a ubiquidade ou onipresença (do latim, ubique, por todo lado). Ou seja, quando um documento, seja na forma de textos, imagens ou som, é virtualizado no ciberespaço, torna-se potencialmente disponível para todos, ao mesmo tempo (Levy, 2011).

Uma segunda característica do ciberespaço, é que todos os documentos encontram-se virtualmente interconectados. Essa interconexão ocorre por meio de links que permitem ligações e redirecionamentos entre sítios distintos da internet, resultando na produção, pelo usuário, de um hipertexto próprio, baseado nas escolhas feitas por este no momento da navegação (Levy, 2011).

Uma terceira característica é o automatismo manipulador de símbolos, ou seja, a presença de robôs que são capazes de agir sobre os símbolos, de calcular, de computar. São capazes de fazer operações aritméticas e lógicas e, a partir dessas operações aritméticas-lógicas, realizam toda sorte de operações, ou seja, operações de imagens, de sons etc. Os documentos em questão que estão no ciberespaço são animados desde o interior por robôs e podem ser acessados por todos mediante a interatividade. Os usuários podem, portanto, acessar e modificar facilmente estes dados interativos (Levy, 2011).

Ubiquidade, interconexão e animação pelos robôs manipuladores de símbolos, reunidos em uma memória numérica única mundial, irão conduzir o processo de evolução da inteligência artificial. Contudo, o que é AI? O Léxico Oxford descreve IA como a tecnologia usada para automatizar tarefas que normalmente requerem inteligência humana. Esse tipo de conceituação relaciona IA à automatização de tipos de tarefas especificas realizadas pela inteligência humana. O maior sucesso dessa espécie de tecnologia deu-se na aplicação da automatização de atividades complexas como o jogo de cartas, tradução de línguas e a condução de veículos.

Afinal, o que torna a AI diferente dos processos de automatização em geral? Na verdade, a IA compartilha uma característica comum com a inteligência humana, pois quando as duas inteligências realizam certas atividades, usam alto níveis 
de processos cognitivos associados. Essa definição pode levar a pensar que IA é um sistema de "máquinas pensantes" (Surden, 2014), ou de produção de resultados pelo uso de cognição informática sintética que sobre-passa a inteligência humana. A verdade, contudo, é que o sistema de IA não é uma máquina pensante inteligente.

De fato, os resultados produzidos pelo AI são alcançados, na maioria das vezes, de forma heurística, mediante o uso de padronização, regras e informações que foram computacionadas. Para chegar aos resultados em tarefas com alto nível de complexidade, a AI, como sistema interdisciplinar, incorpora ideias, técnicas e pesquisas de campos do conhecimento variados, incluindo, estatística, linguística, robótica, engenharia elétrica, matemática, neurociência, economia, lógica, filosofia, dentre outras.

Então, não sendo a IA uma reprodução da inteligência humana, de que se trata? Que tipo de mecanismo permite a AI automatizar suas tarefas de alta complexidade? Atualmente, há duas abordagens principais quanto à tecnologia de inteligência artificial: marchine learning e a logical rules and knowledge representation. Essa são as duas maneiras de programar os sistemas de computadores para realizar tarefas de AI. A tecnologia da machine learning está relacionada à aprendizagem de máquinas, na qual o sistema depende de um programa que identifique padrões em dados para tomadas de decisões inteligentes. A teconologia da logical rules and knoledge representation, por sua vez, envolve a representação de conhecimento e regras lógicas, na qual se modela um fenômeno real ou processo em linguagem computacional com o objetivo de automatizar as respostas. Ambas categorias de AI podem ser eficazes, a depender do domínio aplicado (Buest, 2017).

Poder-se-ia pensar que o uso da IA em tarefas complexas podem ser generalizados para qualquer tipo de tarefa. Contudo, deve-se deixar claro que a AI tende a ser uma "inteligência" estreita ou limitada, formatada para tarefas específicas e com características particulares. A tecnologia atual da IA não é, portanto, adaptável a qualquer tarefa (Gibney, 2017). Essa lógica distancia-se de perspectivas futuristas, a exemplo do pensamento de Uiara Pereira e Tarcisio Teixeria (2019, p. 124) que prospectam maquinas ultrapassando a capacidade intelectual humana, substituindo-a ou até mesmo controlando-a, com o objetivo de sobrevivência.

Atualmente, a IA tem melhor uso em atividades que possam ser padronizadas, marcadas por regras e de estruturas formais. A tecnologia da IA não tem um uso generalizado para problemas que envolvam conceitos abstratos, razoabilidade e entendimentos de palavras. As tecnologias de automatização não alcançam os problemas que requerem senso comum, julgamento ou intuição (Surden, 2019, p. 1308). Compreender os limites e as possibilidade da IA é fundamental para entender qual a atual potencialidade de sua aplicação na ciência jurídica e, consequentemente, na construção da norma decisória para solução de litígios.

\section{O Processo de Construção da Norma Decisória}

Propor uma relação entre Direito e IA revela-se, para alguns, como a possibilidade de uma revolução na forma de atuar com o Direito. Para entender todas as consequências dessa relação, importa aprender essas duas áreas de conhecimento. No item anterior, abordou-se o conceito, as possibilidades e os limites da IA. A pergunta que se segue, em vista da relação aqui proposta, é: qual o objeto da ciência jurídica ou do que trata o Direito? Pode ser dito que a ciência jurídica tem por objeto a norma jurídica, sendo esta a forma de manifestação do Direito. A resposta, entretanto, apenas desloca o problema para o âmbito da norma jurídica, pois, dependendo da abordagem epistemológica, as normas como instrumento fundamental de aplicação do Direito podem entendidas por uma perspectiva sociológica, positivista normativista ou pós-positivista.

Para a sociologia jurídica, as normas encontram-se consubstanciadas na adoção de condutas, praticadas de forma reiterada e com sentimento de sua obrigatoriedade, pelos membros de uma determinada comunidade. Já na concepção positivista normativista, a norma é uma previsão abstrata obtida através da interpretação de textos jurídicos, sem qualquer vinculação com a realidade. Visando superar o vácuo existente entre essas duas concepções, Friederich Muller (2008), em uma 
perspectiva pós-positivista contemporânea, propõe que a norma jurídica resulta da interação dialética entre texto normativo e elementos da realidade, os quais funcionam como elementos estruturantes da norma.

No desenvolvimento de sua teoria estruturante da norma jurídica, Müller (1999) distingue texto normativo e norma, sendo esta o resultado do trabalho do jurista guiado pelo caso concreto. O método concretizante, portanto, rejeita a identificação entre norma e texto (Xerez \& Rocha, 2019).

O "programa normativo", obtido através da interpretação dos textos normativos é apenas um dos elementos necessários ao processo de construção da norma jurídica, a qual depende, em igual medida, do "âmbito normativo", ou seja, dos elementos da realidade juridicamente relevantes. A identificação do "programa normativo" e do "âmbito normativo" ocorre diante do caso concreto, em uma relação dialática, cujo resultado é a produção da norma decisória. Essa nova compreensão, demonstra a reciprocidade complementar entre normatividade. O texto normativo não é completo, mas apenas enuncia uma expectativa de sua plausibilidade à luz do contexto prático, sendo apenas o iceberg de uma prescrição juspositiva (Müller, 1999).

A estrutura articulada da norma realiza um duplo movimento por meio da interação entre "programa normativo" e "âmbito normativo", cujo resultado é a norma decisional a ser aplicada ao caso concreto. A relação complementar e comunicativa entre texto normativo e realidade produz a norma jurídica entendida como sendo a norma de decisão.

A teoria estruturante proposta por Muller permite abandonar o mito de que a norma jurídica é construída a partir de textos positivados, mediante operações lógico-formais desvinculadas da realidade, com vistas à uma pretensa reconstrução objetiva da legalidade consubstanciada em uma norma geral e abstrata. A teoria estruturante demonstra que a norma jurídica é essencialmente a norma decisória, cuja construção depende, em igual medida, de elementos textuais e elementos da realidade, interconectados em uma relação dialética.

\section{Os Impactos Possíveis da Inteligência Artificial no Direito}

Após entender os limites e possibilidades reais da IA nessas duas primeiras décadas do século XXI, bem como compreender o processo de interpretação e aplicação do Direito envolvido na construção da norma decisória, resta analisar que impacto pode ter a inteligência artificial na construção norma decisória.

A resposta a esta pergunta exige a tarefa de fazer dialogar a IA, nos modelos marchine learning e logical rules and knoledge representation, e o complexo modelo de inteligência humana envolvido no processo de concretização de uma norma de decisão. Tendo discorrido sobre estes sujeitos do diálogo nos itens anteriores, pode-se concluir que a AI, não obstante possa contribuir positivamente para o processo de concretização da norma de decisão, não é capaz de realizar de forma autônoma tal processo em razão, notadamente, de seus limites para elaboração de juízos axiológicos. A prática jurídica é pensada para habilidades cognitivas avançadas e que tal cognição permanece fora das habilidades de cognição da tecnologia da IA corrente. De fato, os algoritmos contemporâneos do IA não possuem a capacidade de replicar a maioria das habilidades intelectuais humanas e, por conseguinte, realizar, de forma autônoma, o processo de concretização da norma de decisão.

Uma IA que envolvesse thingking marchines com habilidades que pudessem superar o nível de cognição da inteligência humana, chamada de Inteligência artificial geral (Artificial General Intelligence - AGI), permanece ainda como aspiração científica (Mills, 2018). Na verdade, não passa de ficção das indústrias de entretenimento que apresentam computadores envolvidos em diálogos filosóficos ou operando sistemas cognitivos independentes (Ebiri, 2015). Atualmente, os sistemas de IA não estão desenhados para atuar nos altos níveis de habilidade humana necessários ao processo de construção da norma decisória, os quais exigem raciocínio abstrato-valorativo para manutenção do diálogo entre o programa da norma e o âmbito da norma. 
Delineados os limites reais da IA diante da matriz de cognição exigida pelo Direito, pode-se tomar uma posição mais realista quanto os possíveis impactos daquela sobre a construção da norma de decisão. Nesse sentido, desde 1987, o mundo científico tem realizado a Conferência Internacional de Inteligência Artificial e Direito (International Conference Artficial Intelligence and Law - ICAIL), na tentativa de avançar na aplicação das tecnologias de IA ao Direito. Em 2000, as pesquisas em IA e Direito deixaram de centrar nas técnicas de representação de conhecimento, para migrar para técnicas de machine learning. Muitas das aplicações da IA na área do Direito tem surgido do empreendedorismo de Startups, com a finalidade de tornar mais eficiente a aplicação do Direito, o que pode trazer ganhos para o processo de construção da norma decisória em razão de recursos de algoritmos que possibilitam a seleção de dados tais como textos legais, estatísticos, dentre outros.

Diversos outros avanços estão sendo implementados por Centros Interdisciplinares de pesquisas de engenharia jurídica universitária, como é o caso do Centro CodeX da Stanford. Deve-se considerar ainda o trabalho de vanguarda realizado pela Faculdade de Direto da Universidade de Montréal, com o seu laboratório de Cyberjustice. Fundado em 2010, pelo prof. Karim Benyekhlef, o laboratório trata de conectar as ciências jurídicas com a informática. Para Benyekhlef, a Cyberjustice é a integração das tecnológicas algorítmicas, da informação e da comunicação no interior dos processos judiciários e extrajudiciários de resolução de conflitos. Seu objetivo é favorecer o acesso e diminuir os custos da justiça (Daniel, 2018). No laboratório, pesquisadores analisam as expectativas dos cidadãos, dialogam com juízes e advogados, e, após, modelam e desenham softwares para auxiliar os litigantes. Este trabalho é baseado nas novas tecnologias da "aprendizagem profunda" (deep dearning) e de aprendizado automático (machine learning), em estreita colaboração com a Montreal Institute of Learning Algorithms (MILA), liderado por Yoshua Bengio.

Um dos últimos projetos do Laboratório Cyberjustice é o agente conversacional (Chatbot) destinado ao direito de locação. O programa avalia a validade de uma planta, analisa a jurisprudência e redige contratos claros e acessíveis. Outro programa revolucionário é o PARLe (Plataforma de ajuda na resolução de litigância eletrônica), uma plataforma de resolução de conflito online provida pela Secretaria de proteção ao consumidor de Quebec. O programa viabiliza que os consumidores promovam suas reclamações contra comerciantes ou empresas, permitindo que os mesmos possam entrar em contrato direito para negociação. No caso de desacordo, um mediador intervém pelos mesmos meios virtuais. Conforme dados, sem esse recurso, os consumidores teriam muitas vezes abandonado suas reivindicações em razão dos danos serem pequenos e os procedimentos longos e custosos. Agora, estas disputas são resolvidas em média vinte e oito dias em vez de doze meses no Tribunal. Finalmente, se a mediação não funciona, o Cyberjustice oferece o primeiro Tribunal inteiramente online para favorecer a negociação e mediação de conflitos.

No Brasil, o projeto de pesquisa e desenvolvimento de aprendizado de máquina sobre dados judiciais das repercussões gerais do Supremo Tribunal Federal, em parceria com os cursos de Direito, Engenharia de Software e Ciências da Computação da Universidade de Brasília, nomeado de Victor, aplica métodos logaritmos com o objetivo de reconhecer padrões nos processos jurídicos relativos a julgamentos do STF. Maia Filho e Junquilho (2018, pp. 225-226) faz ver que o objetivo do projeto não é que o algoritmo tome a decisão final acerca da repercussão geral, mas sim que, com as maquinas treinadas para atuar em camadas de organização dos processos, os responsáveis pela análise dos recursos possam identificar os temas relacionados de forma calara e consistentes.

Longe dos antigos templos, das pilhas de papéis sobre as mesas dos juízes e das provas acumuladas nos arquivos, o mundo da Justiça está em meio a uma revolução. Pretende-se a criação de um Direito SMART, ou seja, Scientific (científico), Mathematical (matemático), Algorithmic (algorítimico), Risk-driven (dirigido pela análise de riscos), e Technological (tecnológico). Na Suécia e na Estónia, os títulos de propriedade são agora trocados por meio de blockchains, extinguindo as taxas notariais. Nos Países Baixos, já existe uma plataforma online apoiada pelo Estado para resolver divórcio. Uma forma mais rápida, mais barata e menos passional do que o Tribunal, como alegam os seus designers fundadores. Na Grã-Bretanha, 
um algoritmo fiscal acompanha os contribuintes, calcula os seus rendimentos e despesas em tempo real e monitoriza os seus sinais externos de riqueza. Gérald Darmanin, Ministro da Ação e das Contas Públicas, revelou no final de 2018, que será experimentado um sistema semelhante na França no início de 2019 (Haddad, 2018).

A tecnologia usada atualmente atende e está adaptada à resolução de conflitos de baixa intensidade. Seu uso para conflitos complexos ainda é incerto, especialmente para aqueles casos que envolvem juízos axiológicos a serem elaborados diante de situações fáticas não comuns. Nesse sentido, basta pensar em questões correcionais ou criminais, na quais o perigo de delegar decisões a uma tecnologia é considerável. Nos Estados Unidos, um algoritmo judicial para prever o risco de reincidência produziu uma discriminação racial e social evidente (Naughton, 2019).

Essa realidade confirma o dito anteriormente, pois, ainda que os avanços sejam considerados e as contribuições da AI para a aplicação do Direito sejam significativas, haverá de se esperar algumas gerações para que a ontologia informacional complete seu ciclo na relação entre IA e produção de normas decisórias.

\section{Considerações Finais}

Percebe-se, mediante considerações abordadas durante o texto, que não se trata de uma superinteligência capaz de superar as capacidades cognitivas da inteligência humana. Pelo contrário, a IA produz resultados a partir da utilização de padrões e regras que lhe permitem produzir recursos úteis em contextos delimitados.

Exatamente por essas características conceituais, a tecnologia da IA tem limites, sobretudo, em razão de sua pouca habilidade de manipular abstrações, ponderar valores e tomar decisões. O complexo processo de construção de uma norma de decisão, com suas tarefas de hermenêutica do texto normativo, diálogo com elementos da realidade e inafastáveis juízos axiológicos, é atualmente inalcançável à IA.

A compreensão dos limites da AI, ao invés de romper o diálogo com o Direito, restitui esta relação a um contexto possível. A maioria das tarefas onde a IA provou ser bem-sucedida envolve áreas altamente estruturadas com base em respostas do tipo certo-errado e padrões subjacentes que podem ser algoritmicamente detectadas. É exatamente nesse campo que a inteligência algorítmica pode ajudar aos atores do Direito na concretização da norma de decisão, na medida em que padroniza buscas por temas em jurisprudência, dados estatísticos, dentre outros elementos objetivos. Com esse tipo de ajuda da máquina, a prática jurídica e a administração da Justiça tenderão a se tornar mais eficazes, claras e econômicas.

\section{Referências}

Bôas Filho, O. V. (2010). Tendências da análise antropológica do direito: algumas questões a partir da perspectiva francófona. Revista de Direito GV, São Paulo, 6(1) p. 321-328, Jun./Jan.

Buest, R. (2017). Artificial intelligence is about machine reasoning or who machine learning is just a fancy plugin (CIO, 03 nov. 2017). https://www.cio.com/article/3236030/artificial-intelligence-is-about-machine-reasoning-or-when-machine-learning-is-just-a-fancy-plugin.html.

Conselho Nacional de Justiça. Justiça em números. www.cnj.jus.br/pesquisas-judiciarias/justica-em-numeros/.

Conselho Nacional de Justiça. Política Nacional de tratamento adequado de conflitos. Resolução no. 125 de novembro de 2010. https://atos.cnj.jus.br/atos/detalhar/atos-normativos?documento=156.

Daniel, Sara. L’OBS. (2018). Intelligence artificielle: Karim Benyekhlef, l'homme qui "automatise" la justice. www.nouvelobs.com.

Ebiri, B. (2015). The 15 best robot movies of all time. www.vulture.com.

Gibney, E. (2017). Self-taught ai is best yet at strategy game go.www.nature.com/news/self-taught-ai-is-best-yet-at-strategy-game-go-1.22858.

Haddad, M. P. (2018). Évasion fiscale : les comptes des Français sur les réseaux sociaux bientôt scrutés. TTL. www.rtl.fr.

Leiner, B. M. (1999). A brief history of the intenet . www.arxiv.org/html/cs/9901011.

Levy, P. (2011). La sphère sémantique. Computation, cognition, économie de l'información. Tome 1, Universitè du Ottawa, p. 63.130. 
Research, Society and Development, v. 10, n. 8, e3710815361, 2021

(CC BY 4.0) | ISSN 2525-3409 | DOI: http://dx.doi.org/10.33448/rsd-v10i8.15361

Mcevoy, M. (2019). Analysis: 2019 legal tech investments top \$ 1B after stong Q3. https://news.bloomberglaw.com.

Maia Filho, M. S., \& Junquilho, T. A. (2019). Projeto Victor. Perspectivas de aplicação da inteligência artificial ao direito. Revista de Direitos e Garantias Fundamentais, Vitória, 19(3), set./dez.

Mills, T. (2018). AI vs AGI: What's the difference?. www.forbes.com.

Müller, F. (2008). Teoria estruturante do direito. Revista dos Tribunais.

Müller, F. (1999). Métodos de trabalho do direito constitucional. Síntese.

Naughton, J. (2016). Even algorithms are biased against black men. www.theguardian.com.

Pereira, U. V., \& Teixeira, T. (2019). Inteligência artificial. A quem atribuir responsabilidade? Revista de Direitos e Garantias Fundamentais, Vitória, 20(2), mai/ago.

Privovarov, V. (2019). 713\% growth: legal tech set an investment record in 2018. www.forbes.com.

Rosa, C., \& Spaler, M. (2019). Experiências privadas de ODR no Brasil. Revista Jurídica da Escola Superior de Advocacia da OAB-PR, 3(3), dez.

Surden, H. (2019). Artificial intelligence and law. An overview. Georgia State Universty law Review, 35(4), Summer.

Surden, H. (2014). Machining learning and law. Washington Law Review, v. 87.

Xerez, R., \& Rocha, K. (2019). Análise jurídica da poliafetividade a partir do filme Eu Tu Eles. Anamorphosis RDL, 5(1), jan.-jun. 